\title{
Wearable Vibrotactile Interface Using Phantom Tactile Sensation for Human-Robot Interaction
}

\author{
Julian Seiler ${ }^{1}$, Niklas Schäfer ${ }^{1(\otimes)}\left(\mathbb{D},{\text { Bastian } \text { Latsch }^{1}(\mathbb{D}) \text {, Romol Chadda }}^{1}\right.$, \\ Markus Hessinger $^{1}$, Philipp Beckerle ${ }^{2,3} \mathbb{D}$, and Mario Kupnik ${ }^{1}$ \\ 1 Technische Universität Darmstadt, Measurement and Sensor Technology, \\ Merckstraße 25, 64283 Darmstadt, Germany \\ schaefer@must.tu-darmstadt.de \\ 2 Technische Universität Dortmund, Robotics Research Institute, \\ Otto-Hahn-Straße 8, 44227 Dortmund, Germany \\ 3 Technische Universität Darmstadt, Institute for Mechatronic Systems, \\ Otto-Berndt-Straße 2, 64287 Darmstadt, Germany
}

\begin{abstract}
We present a wearable vibrotactile feedback device consisting of four linear resonant actuators (LRAs) that are able to generate virtual stimuli, known as phantom tactile sensation, for human-robot interaction. Using an energy model, we can control the location and intensity of the virtual stimuli independently. The device consists of mostly 3Dprinted rigid and flexible components and uses commercially available haptic drivers for actuation. The actuators have a rated frequency of 175 $\mathrm{Hz}$ which is close to the highest skin sensitivity regarding vibrations (150 to $300 \mathrm{~Hz}$ ). Our experiment was conducted with a prototype consisting of two bracelets applied to the forearm and upper arm of six participants. Eight possible circumferential angles were stimulated, of which four originated from real actuators and four were generated by virtual stimuli. The responses given by the participants showed a nearly linear relationship within $\pm 10^{\circ}$ for the responded angle against the presented stimulus angle. These results show that phantom tactile sensation allows for an increase of spatial resolution to design vibrotactile interfaces for human-robot interaction with fewer actuators.
\end{abstract}

Keywords: Vibrotactile feedback $\cdot$ Phantom tactile sensation • Human-robot interface

\section{Introduction}

Human-robot interaction (HRI) becomes more and more common due to progress in fields like robotics and artificial intelligence but also psychology. In particular, when a human and a robot are working on a common task, a bidirectional transfer of information is required $[1,2]$.

Usually, the visual channel is already in use. Therefore, vibrotactile feedback can be helpful when added to provide additional information [3], especially

(C) The Author(s) 2020

I. Nisky et al. (Eds.): EuroHaptics 2020, LNCS 12272, pp. 380-388, 2020.

https://doi.org/10.1007/978-3-030-58147-3_42 
when multiple tasks are being performed and the workload is high [4]. Vibrotactile feedback devices can be used to present physical information, e.g., contact location, as well as abstract information, e.g., direction. They have been investigated in various applications such as robotic teleoperation [5], spatial awareness in virtual reality [6], navigation [7], and motion guidance [8].

Most collaborative tasks in the context of HRI, e.g., handling a tool or carrying an object, are performed by the human using hands and arms. Thus, vibrotactile feedback to the human arm offers a possibility to provide intuitively understandable information. In particular, circumferential feedback, i.e., feedback at different locations around the arm, enables providing information from different directions.

There are many vibrotactile feedback devices in research [9], including some for feedback around the arm [10-12]. Most of them use eccentric rotating mass motors (ERMs) due to the simplicity of control, small form factor, and low cost. However, the inherent coupling of amplitude and frequency of an ERM can be a limitation because the perceived intensity of a vibrotactile stimulus depends not only on its amplitude but also on its frequency [13].

A commercially available vibrotactile feedback device using ERMs is VibroTac (SENSODRIVE, Weßling, Germany). It was originally developed at the German Aerospace Center for the application on the human arm [12]. Due to the ergonomic design, it can be worn on a wide range of arm circumferences. It provides vibrotactile stimuli at six circumferential locations.

The spatial resolution of a vibrotactile feedback device can be increased by utilizing tactile illusions [14]. In [15] an illusion known as phantom tactile sensation was used to induce vibrotactile cues at any circumferential location around the wrist with six ERMs. Phantom tactile sensations can be classified regarding the perceived stimulus either being stationary or moving across the skin [16].

In this paper, we present a wearable vibrotactile feedback device for the human arm consisting of two bracelets. We investigate the feasibility of generating vibrotactile cues at eight circumferential locations around the arm with only four actuators by using stationary phantom tactile sensation. The application of linear resonant actuators (LRAs) ensures a constant frequency for all vibration amplitudes. The modular feedback device is evaluated experimentally on the forearm and the upper arm.

\section{Fundamentals}

The occurrence of phantom tactile sensations in haptics, not to be confused with phantom limb illusions, was first described by von Békésy in 1957 [17]. It terms the phenomenon that two simultaneous vibrotactile stimuli produced by two closely spaced actuators are perceived as one single vibration in between (Fig. 1a). This effect is based on sensory funneling [18]. Location and intensity of the phantom tactile sensation can be controlled by the intensities of the actuators. The location results from the actuators' relative magnitudes, whereas the intensity can be controlled by the actuators' absolute magnitudes. Two 


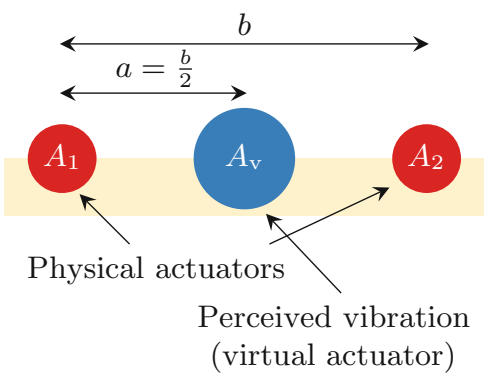

(a)

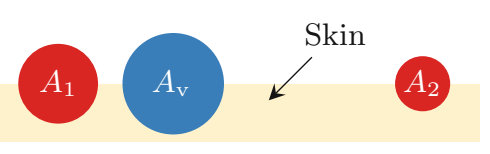

(b)

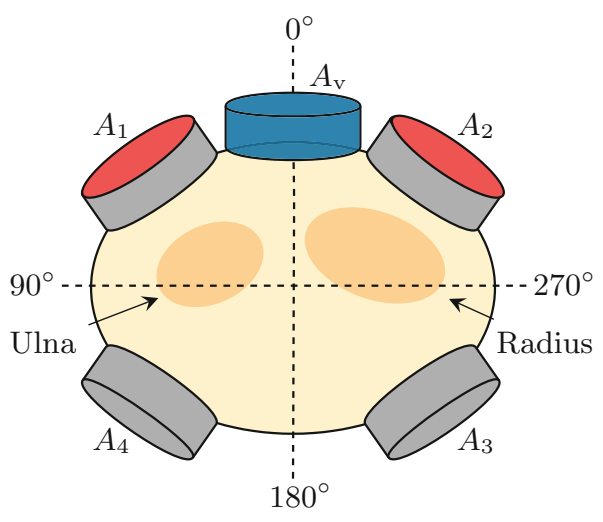

(c)

Fig. 1. Basic principle of phantom tactile sensation and placement of the actuators. The phantom tactile sensation is based on sensory funneling. Two simultaneous vibrotactile stimuli produced by two closely spaced actuators are perceived as one single vibration in between (a). Adjusting the actuators' vibration intensities allows shifting the perception closer to the actuator with higher magnitude (b). In order to provide directional cues, four LRAs are arranged equidistant around the left human forearm (c). A phantom tactile sensation is created by activating two adjacent actuators. Location and intensity of the virtual actuator are controlled based on an energy model [19].

vibrations with equal intensities result in a centered phantom tactile sensation. Adjusting the magnitudes equally leads to a sensation with the same location but different intensity. If the intensities are different, the phantom tactile sensation is located closer to the actuator with the higher magnitude (Fig. 1b).

The energy model proposed in [19] allows controlling the relative location $\beta=\frac{a}{b}$ and the intensity $A_{\mathrm{v}}$ of a virtual actuator induced by phantom tactile sensation independently. The required intensities of the two physical actuators are

$$
A_{1}=\sqrt{1-\beta} \cdot A_{\mathrm{v}}, \quad A_{2}=\sqrt{\beta} \cdot A_{\mathrm{v}} .
$$

This energy model is based on two assumptions. First, the vibration frequencies of both actuators are equal. Second, the skin sensitivity thresholds at the locations of the physical actuators are identical.

\section{Design and Construction}

In order to provide directional vibrotactile feedback, four LRAs (G1036002D, Jinlong Machinery \& Electronics, Wenzhou, China) are arranged equidistant 
around the arm (Fig. 1c). Unlike ERMs, the vibration amplitude of an LRA can be adjusted without changing the vibration frequency. This satisfies the energy model's first assumption of equal vibration frequencies. With $175 \mathrm{~Hz}$, the rated frequency of the LRAs is close to the highest sensitivity of the Pacinian corpuscles, which is usually found between 150 and $300 \mathrm{~Hz}$ [20,21].

The feedback device is designed to be wearable as a bracelet on the arm. It consists of multiple 3D-printed segments, which are mounted on an elastic cord (Fig. 2). There are two types of segments. The actuator segments consist of a rigid basis (polylactic acid) and a comparatively flexible mounting (thermoplastic polyurethane, shore hardness $98 \mathrm{~A}$ ) for an LRA which aims at reduction of vibration propagation into the mechanical structure. The intermediate segments carry the electronics as well as the control unit and are used for cable routing. The alternating arrangement of the segments creates a zigzag pattern, which increases the overall elasticity and an equidistant actuator arrangement [12].

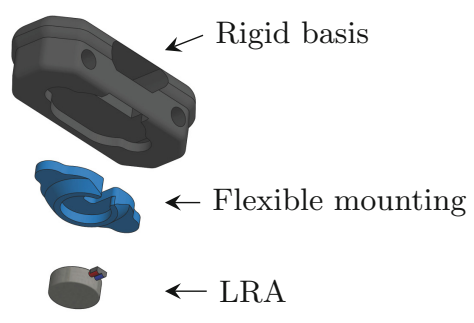

(a) Actuator segment

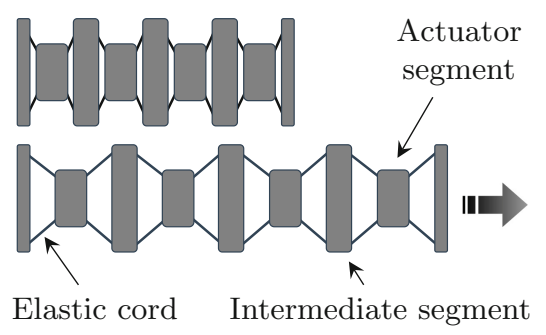

(b) Structure of the vibrotactile bracelet

Fig. 2. Each actuator segment consists of a rigid basis, a flexible mounting, and an LRA (a). Actuator segments and intermediate segments are arranged alternating on an elastic cord (b). The zigzag pattern of the elastic cord ensures equal distances between the actuators in relaxed and stretched state [12].

The LRAs require a sinusoidal driving voltage at their resonance frequency. We use commercially available haptic drivers (DRV2605L, Texas Instruments, Dallas, Texas, U.S.) in combination with an ESP32-WROOM-32 module (Espressif Systems, Shanghai, China) controlling the amplitudes. The control unit receives commands containing direction and magnitude from a PC via Bluetooth. The desired vibrotactile cues between two physical actuators are generated by a control algorithm that applies the energy model (Eq. 1).

\section{System Evaluation}

The goal of our evaluation experiment was to investigate the perceived direction of vibrotactile cues generated by the developed prototype (Fig. 3a). The locations of the vibrotactile cues resulted either from one real actuator at its own location or from two actuators by inducing phantom tactile sensation in between. Both, 
forearm and upper arm, were stimulated with vibrotactile cues. In our first test, six participants ( 1 female, 5 male, $22.7 \pm 1.6$ years) gave prior informed consent and took part in the experiment. They were informed that their vibrotactile perception is investigated in the experiment but no information about phantom tactile sensation and placement of the actuators was given.

After measuring the circumferences of the forearm $(25.8 \pm 3.3 \mathrm{~cm})$ and the upper arm $(30.8 \pm 2.3 \mathrm{~cm})$, the participants were requested to wear the system on the left arm. The vibrotactile bracelets were placed in the middle of the respective arm segment, ensuring an equidistant arrangement of actuators. The origin of the reference frame for each arm segment was defined to be collocated with the driver electronics. The bracelet with the control unit and the battery was always worn on the upper arm. The elastic cord was adjusted to the individual arm circumference of each participant in order to ensure that the vibrotactile bracelets could be worn comfortably.

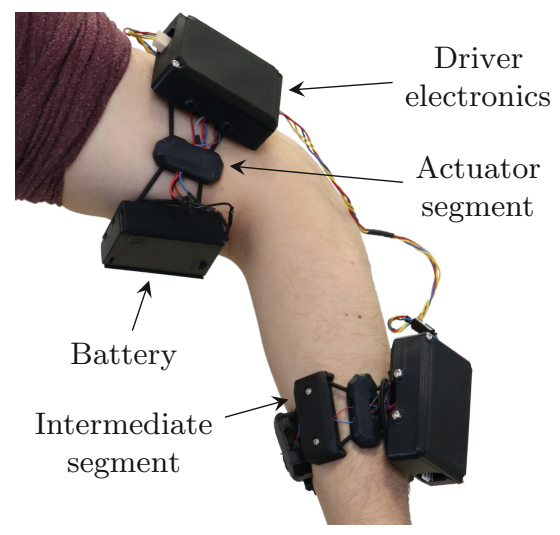

(a)

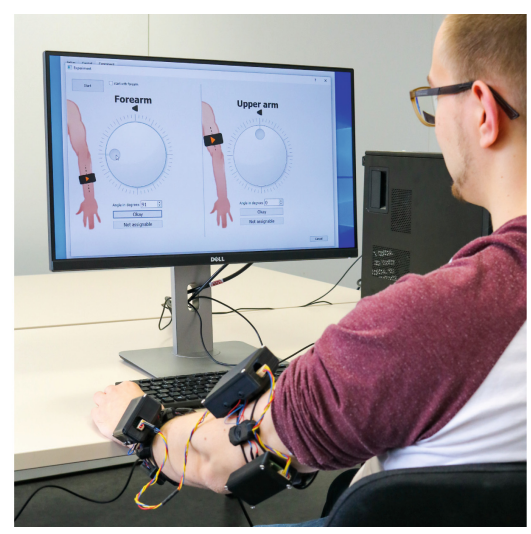

(b)

Fig. 3. Prototype of the vibrotactile feedback device consisting of two bracelets worn on the forearm and the upper arm (a). The bracelet on the upper arm contains the control unit (hidden behind the upper arm), the driver electronics, and the power supply. Due to the modular system architecture, up to eight devices with up to eight actuators each can be connected. During the evaluation experiment, the subjects were asked to adjust the rotary knob to the perceived stimulus angle (b).

\subsection{Experimental Procedure}

The participants were seated in front of a computer screen with a graphical user interface (GUI) consisting of two rotary knobs for an intuitive selection of the vibrotactile cue direction at the forearm and the upper arm, respectively (Fig. 3b). Each arm segment was stimulated with 32 vibrotactile cues. These vibrotactile cues pointed in one of eight possible directions $\left[0^{\circ}, 45^{\circ}, 90^{\circ}, 135^{\circ}\right.$, 
$180^{\circ}, 225^{\circ}, 270^{\circ}$, and $315^{\circ}$ (Fig. 1c)], which were tested four times each in random order. Each vibrotactile cue lasted until a response was given by the participant. The LRAs were driven by a sinusoidal voltage with a frequency of $175 \mathrm{~Hz}$. When stimulating at the location of a real actuator, the rated amplitude of $2.0 \mathrm{~V}_{\mathrm{RMS}}$ was used. In the case of phantom tactile sensation, the amplitudes of the two actuators in use were set such that the intensity of the resulting virtual actuator corresponded to the intensity of one real actuator at rated amplitude (Eq. 1). The participant indicated the direction perceived after each stimulus using the rotary knobs in the GUI (resolution of $1^{\circ}$ ). In addition to the perceived direction, the time for locating the vibrotactile cues was measured as well. After finishing one arm segment, a short break was taken and the participant was requested to rate the difficulty of locating the vibrotactile cues on a scale from 1 to 10 (1 meaning very easy and 10 meaning very hard). The experiment lasted approximately 25 min for each participant.

\subsection{Result and Analysis}

The results of the averaged perceived directions of the vibrotactile cues over the stimulus directions show a nearly linear relationship for both arm segments (Fig. 4). For all but two stimulus angles, the mean response deviates less than $\pm 10^{\circ}$ from the real value. It should be noted though that visual and auditory modalities were not controlled, which may have affected the results.

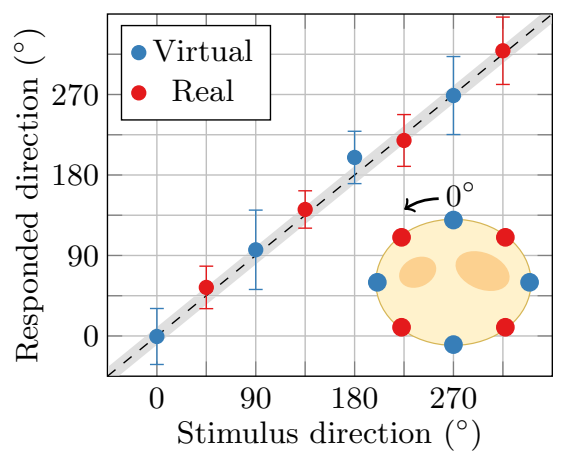

(a)

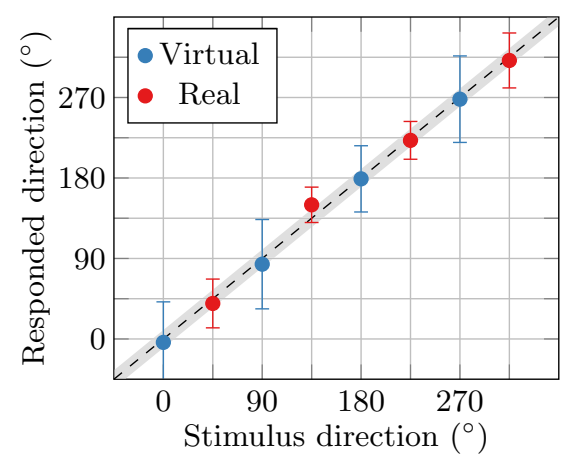

(b)

Fig. 4. Mean values of the responded angles against the presented stimulus angles for the forearm (a) and the upper arm (b). The standard deviations, achieved with the virtual actuators, are higher compared to those observed with the real actuators. For all but two angles, the mean response deviates less than $\pm 10^{\circ}$ (gray band) from the real value. The experiment was always conducted on the left arm (Fig. 1c).

It is noticeable that the perceived vibrotactile cues of the real actuators (forearm $R^{2}=91.81 \%$, upper $\operatorname{arm} R^{2}=93.45 \%$ ) deviate less than those induced 
by virtual actuators (forearm $R^{2}=87.20 \%$, upper arm $R^{2}=83.72 \%$ ) with respect to the ideal linear response. Comparing the results of both arm segments shows that the scattering of the perceived virtually-generated stimuli on the upper arm is stronger than on the forearm. Furthermore, a Wilcoxon signedrank test $(W=0, p=0.0156)$ indicated that the difficulty of locating the vibrotactile cues was rated significantly higher for the upper arm (mean 4.67) in contrast to the forearm (mean 3.17). One possible reason for the latter two observations is the lower innervation density of the mechanoreceptors on the upper arm [22].

In addition to the quantitative results, some of the participants expressed their subjective opinions. They reported that it was difficult to assign the vibrotactile cues using the rotary knobs in the GUI, in particular for the upper arm. This impression is consistent with the higher rating of difficulty for the upper arm. Furthermore, the participants estimated their accuracy of locating the vibrotactile cues between 20 to $30^{\circ}$.

\section{Conclusion and Outlook}

The wearable feedback device developed is capable of generating vibrotactile cues across all circumferential locations around the human arm. This is achieved with only four actuators. In our evaluation experiment the spatial resolution of the vibrotactile feedback can be doubled from four to eight by inducing phantom tactile sensations midway between two actuators. Therefore, we conclude that LRAs with a rated frequency of $175 \mathrm{~Hz}$ are suitable for this type of application although our evaluation experiment satisfies the first assumption of the energy model only.

In future work we will include a determination of skin sensitivity thresholds to check if the second assumption of the energy model is satisfied. At the same time, we plan to apply acoustic and visual shielding to the actuators to gain focus on the vibrotactile cues. For a detailed analysis, further experiments with more participants will be conducted to reinforce the already promising results for applications in HRI, e.g., collision avoidance in teleoperation.

Acknowledgements. This research received support from the Deutsche Forschungsgemeinschaft (DFG) under grants KU 3498/3-1 and KA 417/32-1 within the priority program The Active Self (SPP 2134).

\section{References}

1. Beckerle, P., et al.: A human-robot interaction perspective on assistive and rehabilitation robotics. Front. Neurorobot. 11, 24 (2017)

2. Ajoudani, A., et al.: Progress and prospects of the human-robot collaboration. Auton. Robot. 42, 957-975 (2018)

3. Elliott, L.R., Coovert, M.D., Redden, E.S.: Overview of meta-analyses investigating vibrotactile versus visual display options. In: Jacko, J.A. (ed.) HCI 2009. LNCS, vol. 5611, pp. 435-443. Springer, Heidelberg (2009) 
4. Burke, J.L., et al.: Comparing the effects of visual-auditory and visual-tactile feedback on user performance: a meta-analysis. In: Proceedings of the 8th International Conference on Multimodal Interfaces - ICMI 2006, Banff, Alberta, Canada, p. 108. ACM Press (2006)

5. Bimbo, J., et al.: Teleoperation in cluttered environments using wearable haptic feedback. In: 2017 IEEE/RSJ International Conference on Intelligent Robots and Systems (IROS), Vancouver, BC, pp. 3401-3408. IEEE, September 2017

6. Louison, C., Ferlay, F., Mestre, D.R.: Spatialized vibrotactile feedback improves goal-directed movements in cluttered virtual environments. Int. J. Hum.-Comput. Interact. 34, 1015-1031 (2018)

7. Erp, J.B.F.V., et al.: Waypoint navigation with a vibrotactile waist belt. ACM Trans. Appl. Percept. 2, 106-117 (2005)

8. Bark, K., et al.: Effects of vibrotactile feedback on human learning of arm motions. IEEE Trans. Neural Syst. Rehabil. Eng. 23, 51-63 (2015)

9. Pacchierotti, C., Sinclair, S., Solazzi, M., Frisoli, A., Hayward, V., Prattichizzo, D.: Wearable Haptic Systems for the Fingertip and the Hand: taxonomy, review, and perspectives. IEEE Trans. Haptics 10, 580-600 (2017)

10. Pezent, E., et al.: Tasbi: multisensory squeeze and vibrotactile wrist haptics for augmented and virtual reality. In: 2019 IEEE World Haptics Conference (WHC), Tokyo, Japan, pp. 1-6. IEEE, July 2019

11. Tsetserukou, D., Tachi, S.: Efficient object exploration and object presentation in TeleTA, Teleoperation system with Tactile feedback. In: World Haptics 2009 - Third Joint EuroHaptics conference and Symposium on Haptic Interfaces for Virtual Environment and Teleoperator Systems, Salt Lake City, UT, USA, pp. 97-102. IEEE (2009)

12. Schaetzle, S., et al.: VibroTac: an ergonomic and versatile usable vibrotactile feedback device. In: 19th International Symposium in Robot and Human Interactive Communication, Viareggio, Italy, pp. 670-675. IEEE, September 2010

13. Verrillo, R.T., Fraioli, A.J., Smith, R.L.: Sensation magnitude of vibrotactile stimuli. Percept. Psychophysics 6, 366-372 (1969)

14. Barghout, A., Cha, J., El Saddik, A., Kammerl, J., Steinbach, E.: Spatial resolution of vibrotactile perception on the human forearm when exploiting funneling illusion. In: 2009 IEEE International Workshop on Haptic Audio visual Environments and Games, Lecco, Italy, pp. 19-23. IEEE, November 2009

15. Salazar Luces, J.V., Okabe, K., Murao, Y., Hirata, Y.: A phantom-sensation based paradigm for continuous vibrotactile wrist guidance in two-dimensional space. IEEE Robot. Autom. Lett. 3, 163-170 (2018)

16. Park, G., Choi, S.: Tactile information transmission by $2 \mathrm{D}$ stationary phantom sensations. In: Proceedings of the 2018 CHI Conference on Human Factors in Computing Systems - CHI 2018, Montreal QC, Canada, pp. 1-12. ACM Press (2018)

17. Békésy, G.V.: Sensations on the skin similar to directional hearing, beats, and harmonics of the ear. J. Acoust. Soc. Am. 29, 489-501 (1957)

18. Békésy, G.V.: Funneling in the nervous system and its role in loudness and sensation intensity on the skin. J. Acoust. Soc. Am. 30, 399-412 (1958)

19. Israr, A., Poupyrev, I.: Tactile brush: drawing on skin with a tactile grid display. In: Proceedings of the 2011 Annual Conference on Human Factors in Computing Systems - CHI 2011, Vancouver, BC, Canada, p. 2019. ACM Press (2011)

20. Choi, S., Kuchenbecker, K.J.: Vibrotactile display: Perception, technology, and applications. Proc. IEEE 101, 2093-2104 (2013) 
21. Bolanowski, S.J., Gescheider, G.A., Verrillo, R.T.: Hairy skin: psychophysical channels and their physiological substrates. Somatosens. Mot. Res. 11, 279-290 (1994)

22. Békésy, G.V., Wever, E.G.: Experiments in Hearing. McGraw-Hill, New York City (1960)

Open Access This chapter is licensed under the terms of the Creative Commons Attribution 4.0 International License (http://creativecommons.org/licenses/by/4.0/), which permits use, sharing, adaptation, distribution and reproduction in any medium or format, as long as you give appropriate credit to the original author(s) and the source, provide a link to the Creative Commons license and indicate if changes were made.

The images or other third party material in this chapter are included in the chapter's Creative Commons license, unless indicated otherwise in a credit line to the material. If material is not included in the chapter's Creative Commons license and your intended use is not permitted by statutory regulation or exceeds the permitted use, you will need to obtain permission directly from the copyright holder.

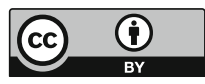

